\title{
THE ISSUES OF SOLUTION APPROXIMATION IN HIGHER-ORDER SCHEMES ON DISTORTED GRIDS
}

\author{
N. B. PETROVSKAYA* \\ School of Mathematics, The University of Birmingham \\ Edgbaston, Birmingham, B15 2TT, UK \\ n.b.petrovskaya@bham.ac.uk \\ A. V. WOLKOV \\ Central Aerohydrodynamic Institute \\ 1 Zhukovsky Street, TsAGI Zhukovsky, Moscow Region \\ 140180, Russian Federation \\ wolkov@progtech.ru
}

Received 26 April 2006

Accepted 30 March 2007

\begin{abstract}
The impact of grid cell geometry on the accuracy of a high order discretization is studied. The issues of solution approximation are investigated on unstructured grids where grid cells are present that are almost degenerate. It will be demonstrated that high-order discretization schemes which employ compact discretization stencil are less sensitive to the geometry of a distorted grid in comparison with those over expanded stencils.
\end{abstract}

Keywords: High-order discretization; unstructured grids; discontinuous Galerkin; leastsquares approximation; distorted geometry.

\section{Introduction}

Over the past three decades computational fluid dynamics (CFD) has successfully evolved into an established tool for the design and analysis for many engineering applications [Darmofal and Haimes (2005)]. On the other hand, the development of CFD methods is still in progress, since the complexity of modern research and engineering problems as well as the rapid increase in computational power require to reevaluate the essential features of CFD codes. One alternative that should be thoroughly explored prior to commitment to built a full three-dimensional simulation tool is the study of high-order discretization schemes [Barth (1998)] to be used in modern CFD codes.

Implementation of high-order schemes is a demanding issue, as they are optimal from a cost reduction point of view for complex CFD problems. Nevertheless,

\footnotetext{
* Corresponding author.
} 
despite the advantages of high-order schemes, by now very few attempts have been made to develop this potentially powerful technology into an industrial CFD code [Venkatakrishnan et al. (2003)]. Most of CFD codes currently use discretization schemes which are second-order accurate in regions where the solution is smooth. Generally, the implementation of high-order schemes for real-life applications is a challenging problem, as it requires a significant computational effort, so that a choice of a discretization scheme should be based on clear understanding of the discretization features.

One crucial issue that should be investigated in detail is the robustness of a high-order discretization scheme. In the present paper we discuss this issue from a geometry viewpoint. Namely, we study the impact of a grid geometry on the quality of high-order approximation on unstructured grids which are heavily exploited in CFD codes to treat complex geometries. It is well known that in numerical solution of CFD problems one may expect computational regions where grid cells almost degenerate. For instance, long and thin grid cells inevitably appear as a result of grid adaptation in boundary layer regions. The quality of the solution approximation on such grid cells may be quite poor, resulting in slow convergence of the approximate solution [Petrovskaya (2001)].

The two discretization approaches which are in the current focus of the research in $\mathrm{CFD}$ include finite volume (FV) and finite element (FE) discretization schemes [Barth (1998)]. Both of these approaches are flexible enough to be used on unstructured grids. Developing a high-order FV scheme often needs a definition of expanded discretization stencils over a computational grid, as increasing the order of the FV scheme requires more accurate gradient approximation which usually involves an expanded stencil. Since the results of a standard gradient reconstruction procedure (i.e. a Green-Gauss or a least-squares reconstruction) depend heavily on the geometry of grid cells, the poor solution approximation may be expected in FV schemes on stretched grids.

A general problem of reconstruction stencils in higher-order FV schemes has been intensively investigated in recent years. The choice of the reconstruction stencils has been discussed in Coirier [1994] where the optimal shape of the stencil has been designed to discretize viscous terms in the Navier-Stokes equations. The approach implemented in Coirier [1994] has been further generalized to 3D problems in Vigneron et al. [2003] where the optimization of weight coefficients at stencil points has been used to keep the discretization scheme consistent. The weighting of stencil points in high-order FV schemes has also been discussed in Barth and Frederickson [1990]; Mavriplis [2003] and Ollivier-Gooch and Van Altena [2002], and it has been demonstrated that in several cases a proper choice of weight coefficients can help to maintain the accuracy of the discretization.

Alternatively, a high-order FE scheme, such as a discontinuous Galerkin (DG) [Bassi and Rebay (1997); Cockburn et al. (2000)] or streamline upwind PetrovGalerkin (SUPG) scheme [Hughes and Brooks (1979)] may be employed to discretize 
the problem. The accuracy of an FE discretization may appear to be sensitive to the shape of grid cells, since the definition of basis functions in the scheme relies upon the grid geometry. However, a crucial difference between high-order FV schemes and FE schemes is that the latter approach employs a compact discretization stencil for solution approximation. The advantage of a compact discretization has been discussed in work [Agarwal and Halt (1999)] where a compact higher-order polynomial reconstruction technique has been developed for numerical solution of the Euler equations on unstructured grids. The results obtained in Agarwal and Halt [1999] for a compact solution reconstruction have been compared with the non-compact scheme in Barth and Frederickson [1990] and the compact discretization appeared to provide better accuracy. However, it was only a simple regular geometry that has been considered in Agarwal and Halt [1999], so that a further study is required to conclude about the efficiency of compact stencils on distorted grids.

In our paper we consider stretched grids to compare the results of the solution reconstruction on compact stencils with those obtained on expanded stencils. We first elaborate a number of simple test cases which demonstrate that compact stencils used for FE schemes are less sensitive to the grid geometry than a scheme on expanded stencils. We then verify the ability of a high-order FE scheme to perform on distorted grids. The examples include an advection equation and a more complicated case of a convection-diffusion equation.

\section{The Expanded and Compact Stencils Over a Distorted Grid}

We begin our consideration of the impact of the grid geometry on the quality of a discretization with a simple test described below. Let us generate a computational grid as follows. Consider a set of grid nodes given by $P_{1}=(0,0), P_{2}=(1+d \alpha, \alpha)$, $P_{3}=(0,1)$ and $P_{4}=(1,1)$. The unstructured grid is generated by subdividing a quadrilateral $D$ with vertices at points $P_{i}, i=1, \ldots, 4$ into two triangles, as it is shown in Fig. 1. The parameter $\alpha \in[0, \infty)$ allows one to control a grid geometry. The value $\alpha=0$ corresponds to a regular geometry of the unit square displayed in Fig. 1(a), while increasing $\alpha$ makes the grid more distorted for a fixed value $d$. A distorted two-cell grid is shown in Fig. 1(b) for $\alpha=1.1$.
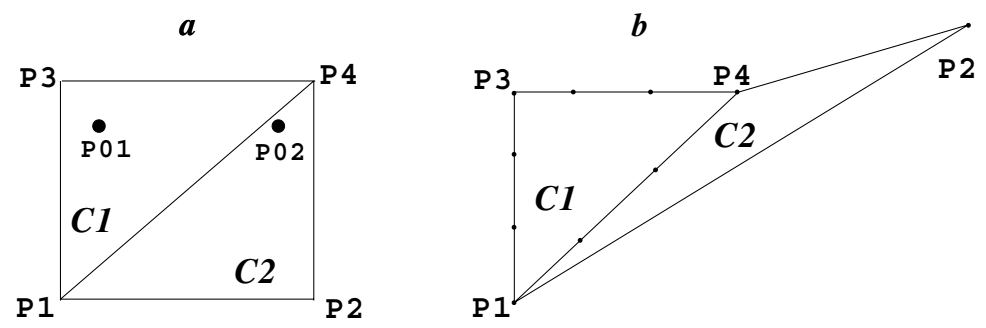

Fig. 1. The test case geometry. The grid distortion is controlled by the parameter $\alpha$. (a) A regular two-cell grid, $\alpha=0$ and (b) a distorted two-cell grid, $\alpha=1.1$. 
We now consider the following advection equation in the domain $D$ :

$$
\frac{\partial u}{\partial x}+a \frac{\partial u}{\partial y}=0
$$

where $a$ is a constant advection velocity. Equation (1) is subject to the boundary condition

$$
u(0, y)=u_{0}(y)
$$

where the function $u_{0}(\xi=y-a x)$ determines the solution to the boundary-value problem (1)-(2),

$$
U(x, y)=u_{0}(y-a x)
$$

Our purpose is to reconstruct the function $U(x, y)$ and the solution gradient $\nabla U=(\partial U / \partial x, \partial U / \partial y)$ at a given point $P_{0} \in D$. Here $\nabla$ is a formal notation for the gradient vector, $\nabla=(\partial / \partial x, \partial / \partial y)$. The question that should be answered as a result of our study is how the geometry of a distorted grid impacts on the accuracy of the approximation. Thus, we intend to reconstruct the quantities above by using expanded stencils over the computational grid and to compare the results with those by the reconstruction on compact stencils.

The reconstruction on expanded stencils will be illustrated by means of the leastsquares (LS) approximation. This approach is related to high-order FV schemes, where the formulation of the scheme requires the evaluation of the solution gradients at grid nodes (e.g. see Mavriplis [2003] and references therein). Alternatively, the reconstruction on a compact stencil will be carried out by the discontinuous Galerkin (DG) discretization scheme. The DG approximation is a high-order FE scheme which exploits piecewise polynomial solution approximation, so that the approximate solution on neighbouring cells is "glued" by means of flux balance equations at grid interfaces Cockburn et al. [2000].

Let us briefly describe the two above-mentioned approaches. The LS reconstruction assumes that the solution function is given at certain points over the domain. Let a data set $\mathbf{U}=\left(U_{1}, U_{2}, \ldots, U_{N}\right)$ be the values of the function $U(x, y)$ at points $\left(P_{1}, P_{2}, \ldots, P_{N}\right)$. We have to fit the data $\mathbf{U}$ to the function

$$
u(x, y)=\sum_{m=1}^{M} u_{m} \phi_{m}(x, y), \quad M \leq N,
$$

where $\phi_{m}(x, y), m=1, \ldots, M$, are basis functions, and the expansion coefficients $\left(u_{1}, u_{2}, \ldots, u_{M}\right)$ are considered as fitting parameters. The polynomial basis functions $\phi_{m}(x, y)$ are given by

$$
\phi_{m}(x, y)=\left(x-x_{0}\right)^{k_{1}}\left(y-y_{0}\right)^{k_{2}},
$$

where $k_{1}+k_{2}=0,1, \ldots, K$, and the $K$ defines the highest polynomial degree of the expansion (4). For any given $K$, the number of basis functions is determined as $M=(K+1)(K+2) / 2$. The point $P_{0}=\left(x_{0}, y_{0}\right)$ is a point in the domain $D$, where the value of the function $u(x, y)$ should be reconstructed. 
The LS approach considers the vector $\mathbf{u}=\left(u_{1}, u_{2}, \ldots, u_{M}\right)$ as the best fit to a given data set, if $\mathbf{u}$ minimizes the following merit function $F^{2}$ :

$$
F^{2}=\sum_{i=1}^{N}\left[\frac{U_{i}-\sum_{m=1}^{M} u_{m} \phi_{m}\left(P_{i}\right)}{\sigma_{i}}\right]^{2},
$$

where $\sigma_{i}$ are the weights of the method. Thus, the parameters $u_{m}$ can be found from the $M$ conditions

$$
\frac{\partial F^{2}}{\partial u_{m}}=0, \quad m=1, \ldots, M,
$$

which are called the normal equations of the method. Taking into account the definition (6), we obtain the normal equations in the following form:

$$
\sum_{i=1}^{N} \frac{1}{\sigma_{i}^{2}}\left[U_{i}-\sum_{j=1}^{M} u_{j} \phi_{j}\left(P_{i}\right)\right] \phi_{m}\left(P_{i}\right)=0, \quad m=1, \ldots, M .
$$

Introducing the weighted data $\mathbf{b}$ and the design matrix $[D]$ as

$$
b_{i}=U_{i} / \sigma_{i}, \quad D_{i j}=\phi_{j}\left(P_{i}\right) / \sigma_{i}, \quad i=1, \ldots, N, j=1, \ldots, M,
$$

the normal equations can be written as $[A] \mathbf{u}=\mathbf{r}$, where the matrix $[A]=[D]^{T}[D]$, and the right-hand side $\mathbf{r}=[D]^{T} \mathbf{b}$. They are to be solved for the vector of parameters $\mathbf{u}=\left(u_{1}, \ldots, u_{M}\right)$,

$$
\mathbf{u}=[A]^{-1} \mathbf{r} .
$$

The definition of the data set $\mathbf{U}$ in the LS methods implies an expanded stencil for the solution reconstruction. For a linear reconstruction $(K=1)$ it is sufficient to define data $U$ at grid nodes, while for a higher-order polynomial degree in the expansion (4) the solution $\mathbf{U}$ should be defined at additional grid points. In our tests, the choice of those points will refer to a regular geometry, as they will be uniformly distributed over the edges of an "undisturbed" grid cell numbered as $C 1$ in Fig. 1. It has been discussed in Petrovskaya [2004] that the LS reconstruction does not take into account the properties of the function $U$, so that the method performs best of all over a uniform grid. Hence, we will only distort the grid by moving the point $P_{2}$ away, while keeping a regular geometry for the rest of the stencil. The data $\mathbf{U}$ will be computed as $U_{n}=U\left(x_{n}, y_{n}\right), n=1, \ldots, N$, where the function $U(x, y)$ is given by solution (3). An example of the LS reconstruction stencil for $K=3$ is given in Fig. 1(b).

Another way to reconstruct a solution or a gradient function at a given point is to solve the boundary-value problems (1) and (2) by a discontinuous Galerkin method. Consider an unstructured computational grid as a set of non-overlapping triangles 
$e_{i}, i=1,2, \ldots, N$. The DG method defines the approximate solution $u_{h}(x, y)$ on each grid cell $e_{i}$ as

$$
u_{h}(x, y)=\sum_{m=0}^{M} u_{m} \phi_{m}(x, y), \quad m=0,1, \ldots, M, \quad x, y \in e_{i} .
$$

Despite the form of the expansion (9) is identical to that in (4), the polynomial basis functions $\phi_{m}(x, y)=\left(x-x_{0 i}\right)^{\alpha}\left(y-y_{0 i}\right)^{\beta}, \alpha+\beta=0,1, \ldots, K$ are now local, they are only defined within the grid cell $e_{i}$. For a cell-centered DG scheme, $x_{0 i}$ and $y_{0 i}$ are the coordinates of the grid cell centroid.

In the DG method a weak formulation of the problem is used to find the approximate solution $u_{h}(x, y)$, the test functions belonging to the same space as the basis functions. Equation (1) is multiplied by test function $\phi_{l}(x, y), l=0,1, \ldots, M$ and is integrated by parts over the cell $e_{i}$. Further substitution of the approximate solution (9) into integrals gives

$$
-\iint_{e_{i}} u_{h}(x, y)\left(\frac{\partial \phi_{l}}{\partial x}+a \frac{\partial \phi_{l}}{\partial y}\right) \mathrm{d} x \mathrm{~d} y+\oint_{\partial e_{i}} h_{u}\left(u^{-}, u^{+}\right) \phi_{l}\left(n_{x}+a n_{y}\right) \mathrm{d} s=0,
$$

where $\mathbf{n}=\left(n_{x}, n_{y}\right)$ is the outward unit normal vector, and the notation $\partial e_{i}$ is used for the boundary of the cell $e_{i}$.

The term $h_{u}\left(u^{-}, u^{+}\right)$in the expression above stands for a numerical flux used to approximate the continuous flux $u(x, y)$ at grid interfaces. The need of the flux approximation in the DG scheme arises from the fact that the approximate solution $u_{h}(x, y)$ is discontinuous at any grid edge. Let $u^{-}$be the solution on the cell $e_{i}$ and $u^{+}$be the solution on the cell $e_{j}$ which shares a given edge with the cell $e_{j}$. For the advection equation (1), the implementation of the Riemann solver, elaborated to discretize the flux $u(x, y)$, results in a well-known definition of the upwind flux,

$$
h_{u}\left(u^{-}, u^{+}\right)= \begin{cases}u^{-}, & \text {if the flow is directed outward from } e_{i}, \\ u^{+}, & \text {otherwise. }\end{cases}
$$

Substitution of the numerical flux (11) into (10) leads to a system of algebraic equations of the form

$$
\mathbf{R}(\mathbf{u})=0
$$

where the vector $\mathbf{R}(\mathbf{u})$ is the residual of the DG method given by (10) on each grid cell and $\mathbf{u}$ is the solution vector. Taking into account the definition of the numerical flux at the left boundary $x=0$ of the domain $D$, the discretization of boundary conditions is straightforward.

It can be seen from definition (10) that the results of the DG discretization on the cell $C 1$ do not depend on the approximate solution on the cell $C 2$. The DG solution on $C 1$ is only defined by the boundary condition at the left boundary $x=0$. Hence, we may expect that, unlike the LS approach (7), a discretization on compact stencils will result in a more accurate solution, as the distortion of the cell $C 2$ does not impact on the solution at the neighbouring cell. Below we compare 
both approximations (7) and (10) for various polynomial degrees in expansions (4) and $(9)$.

\section{Numerical Results on a Model Grid}

In our numerical experiments, the exact solution (3) to the problems (1) and (2) has been chosen as

$$
U(x, y)=(y-a x)^{4} .
$$

The advection velocity is $a=1.0$. Evidently, both LS and DG methods considered for the polynomial degree $K=4$ provide the precise reconstruction of the solution and the gradient at any point of the domain $D$, no matter what the geometry is. Regarding lower polynomial degrees, a robust solution approximation should not be sensitive to the changes in grid geometry. Thus, our first test case is to verify this feature for the solution reconstruction over compact (DG) and expanded (LS) stencils.

Let us vary the parameter $\alpha$ for the fixed value $d=0.7$. We measure the solution and gradient errors at certain grid points. One of our control points, $P_{01}=(0.2,0.75)$, is located in grid cell $C 1$ which geometry remains undisturbed as we increase $\alpha$ (see Fig. $1(\mathrm{~b}))$. Another point $P_{02}=(0.8,0.75)$ belongs to the stretched grid cell $C 2$. We compute the $L_{\infty}$-norm of the solution error as

$$
e_{s}\left(P_{0}\right)=\left|U(x, y)-u_{h}(x, y)\right|_{\mid P_{0}},
$$

at points $P_{01}$ and $P_{02}$. The gradient error is defined as

$$
\begin{aligned}
e_{\nabla}\left(P_{0}\right) & =\left\|\nabla U(x, y)-\nabla u_{h}(x, y)\right\|_{\mid P_{0}} \\
& =\sqrt{\left((\partial U / \partial x)_{\mid P_{0}}-\left(\partial u_{h} / \partial x\right)_{\mid P_{0}}\right)^{2}+\left((\partial U / \partial y)_{\mid P_{0}}-\left(\partial u_{h} / \partial y\right)_{\mid P_{0}}\right)^{2}},
\end{aligned}
$$

where the derivatives of the approximate solution $u_{h}(x, y)$ are obtained by straightforward differentiation of expansion (4) or (9), respectively.

First, we consider the results of the solution and gradient reconstruction for the polynomial degree $K=1$. We use three basis functions in both expansions (4) and (9). For the LS solution reconstruction an expanded stencil is formed by grid nodes $P_{1}$ through $P_{4}$, as it is shown in Fig. 1.

Table 1 displays the solution and gradient error at point $P_{01}$ for the LS and DG reconstructions. It can be seen from the table that the error for the DG method does not depend on the grid geometry, whereas increasing $\alpha$ affects the error of the LS approximation in cell $C 1$. While the DG reconstruction for $\alpha=100.0$ provides a reasonable error in both the solution and the gradient, the LS approximation is unreliable for that value of parameter $\alpha$.

The results of the test for the point $P_{02}$ are presented in Table 2. Now both the DG and LS methods appear to be sensitive to the grid distortion. The error is 
Table 1 . The LS and DG reconstructions at point $P_{01}$. The solution $\left(e_{s}\right)$ and gradient $\left(e_{\nabla}\right)$ errors are shown for the polynomial degree $K=1$.

\begin{tabular}{ccccc}
\hline$\alpha$ & $e_{s}^{L S}$ & $e_{s}^{D G}$ & $e_{\nabla}^{L S}$ & $e_{\nabla}^{D G}$ \\
\hline 0.0 & 0.4375 & 0.1319 & 0.7071 & 0.2357 \\
0.5 & 0.4157 & 0.1319 & 1.1722 & 0.2357 \\
1.0 & 0.3915 & 0.1319 & 1.5970 & 0.2357 \\
10.0 & 0.6832 & 0.1319 & 22.1897 & 0.2357 \\
100.0 & 434.544 & 0.1319 & 7966.24 & 0.2357 \\
\hline
\end{tabular}

Table 2. The LS and DG reconstructions at point $P_{02}$. The solution $\left(e_{s}\right)$ and gradient $\left(e_{\nabla}\right)$ errors are shown for the polynomial degree $K=1$.

\begin{tabular}{ccccc}
\hline$\alpha$ & $e_{s}^{L S}$ & $e_{s}^{D G}$ & $e_{\nabla}^{L S}$ & $e_{\nabla}^{D G}$ \\
\hline 0.0 & 0.5 & 0.1388 & 0.0 & 0.9428 \\
0.5 & 0.7472 & 0.2429 & 0.5399 & 1.4338 \\
1.0 & 0.9655 & 0.3966 & 1.0231 & 2.0713 \\
10.0 & 10.086 & 35.555 & 21.78 & 60.339 \\
100.0 & 3392.8 & 128266.6 & 7965.9 & 28087.22 \\
\hline
\end{tabular}

growing up for both approaches as $\alpha$ is increased resulting in an unreliable reconstruction for relatively small $\alpha=10.0$. Let us notice that the range of parameter $\alpha$ in our tests is adequate to grid geometries appearing in CFD applications. For instance, the high Reynolds number calculations require stretched grids with cell aspect ratio up to $10^{6}$ in a boundary layer. The solution and gradient reconstruction on such cells is a challenging engineering problem (e.g. [see Mavriplis (2003)]) that is still far away from its final solution.

Our next test is to study the convergence properties of both methods. Namely, for a given grid geometry increasing the polynomial degree of the approximate solution should result in a smaller solution and gradient error. While this statement is correct for a regular geometry of a uniform grid [Petrovskaya (2004)], it should be verified on distorted grids. Hence, we increase the polynomial order in approximations (7) and (10) for a given value $\alpha$. Let us point out that, unlike our previous test, we now compute the solution error over the grid. Namely, we measure the $L_{2}$-norm of the solution error over the grid as

$$
e_{L_{2}}=\iint_{D}\left(U(x)-u_{h}(x)\right)^{2} \mathrm{~d} s .
$$

The $L_{\infty}$-norm of the error is defined as

$$
e_{L \infty}=\max _{(x, y) \in D}\left|U(x, y)-u_{h}(x, y)\right|
$$

Error (14) has been computed at Gaussian quadrature points used on each grid cell for numerical integration in the DG discretization (10). 
Table 3. The LS and DG reconstructions for various polynomial degrees $K>0$. The $L_{2}$-norm and the $L_{\infty}$-norm of the solution error are computed over a slightly distorted grid at $\alpha=1.0$.

\begin{tabular}{ccccc}
\hline$K$ & $e_{L \infty}^{L S}$ & $e_{L 2}^{L S}$ & $e_{L \infty}^{D G}$ & $e_{L 2}^{D G}$ \\
\hline 1 & $0.1148 e+1$ & 0.8011 & 0.3085 & 0.1608 \\
2 & 0.6721 & 0.4237 & 0.1552 & 0.1061 \\
3 & $0.4162 e+1$ & $0.3631 e+1$ & 0.0152 & $0.778 e-2$ \\
\hline
\end{tabular}

The results of the test are displayed in Table 3. The parameter $\alpha=1.0$ has been chosen to generate a slightly distorted grid. It can be seen from Table 3 that the value of the solution error is smaller for a given polynomial degree when a DG method is exploited to obtain the approximate solution. This is readily explained by the results of our previous test, as for the DG reconstruction the contribution to the integral error from the first grid cell is quite small. However, the main difference between the two approaches is that increasing the polynomial degree up to $K=3$ reduces the solution error in the DG method, while the cubic solution reconstruction by the LS method results in a solution error greater than that for a linear and quadratic reconstruction. This result well illustrates the problem of the gradient reconstruction over expanded stencils that still remains an open question, given a cell aspect ratio on stretched grids for CFD problems.

\section{High-Order DG Scheme on Distorted Grids}

The results of our tests described in the previous section allow one to conclude that a discretization scheme over a compact stencil may be only locally affected by grid distortion. On the other hand, the ability of the scheme on compact stencils to cope with a distorted geometry should be proven for more complicated test cases. Thus, in this section we further study how a high-order DG scheme performs on distorted grids. The similar study for high-order FV schemes on expanded stencils has been carried out in Petrovskaya [2001].

Let us generate a distorted computational grid to solve the boundary-value problem (1)-(2) in the unit square $\Omega$. The exact solution for the test cases below is chosen as

$$
U(x, y)=\sin (\pi(y-a x))
$$

The grid distortion is now controlled by parameter $\beta \in(0,1)$ as follows. First, a uniform Cartesian grid is generated with a regular distribution of grid nodes given by

$$
x_{i j}=i h_{x}, \quad y_{i j}=j h_{y},
$$




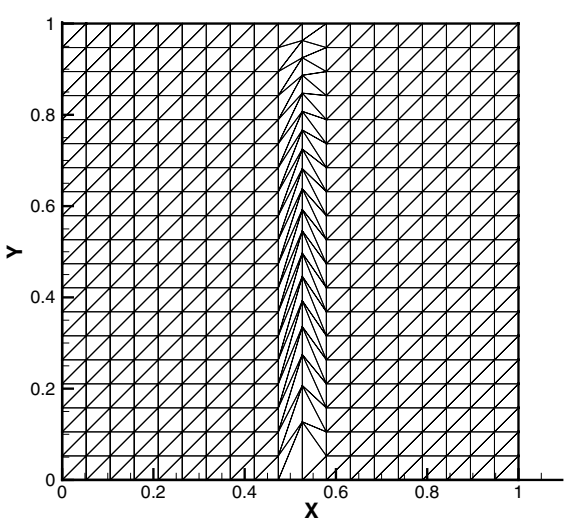

(a)

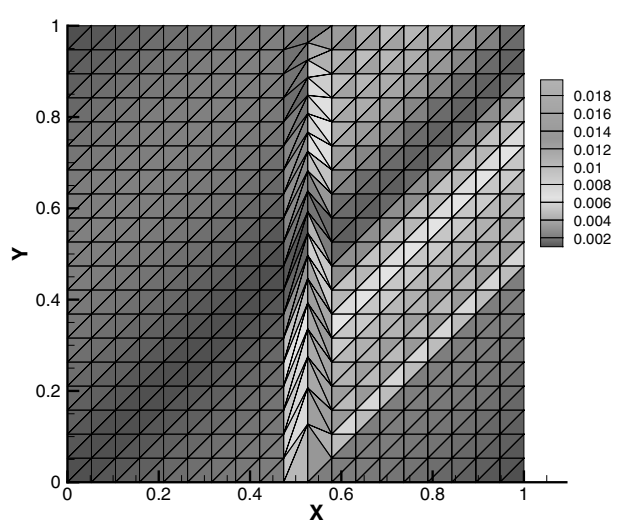

(b)

Fig. 2. (a) An example of a distorted unstructured grid. The distortion parameter $\beta=0.7$. (b) The distribution of solution error (17) for problems (1) and (2) over the distorted grid. The number of grid cells $N_{c}=722$, the distortion parameter $\beta=0.5$.

where $i=1, \ldots, N_{x}, j=1, \ldots, N_{y}, h_{x}$ and $h_{y}$ are grid step sizes in the $x$ and $y$-directions, respectively. Then grid nodes along the selected line $i=i_{0}$ are shifted as

$$
y_{i_{0} j}=\left(j h_{y}\right)^{\beta}, \quad 0<\beta \leq 1,
$$

resulting in a distorted Cartesian grid. The distorted unstructured grid is then obtained by cutting each cell of a structured grid by the diagonal (see Fig. 2).

On the surface, the distorted grids shown in Fig. 2 may look exotic. Apparently, if our goal were to obtain an accurate numerical solution over a computational grid with a given number of grid nodes, a uniform unstructured grid would be more adequate to problem (1)-(2). Our test, however, imitates a wrong grid adaptation in a local domain to study to what extent the DG scheme is sensitive to local grid distortion. Actually, one of the requirements to modern CFD codes is automatic grid generation over a computational domain, [Sherwin and Peiro (2002)]. Furthermore, for many CFD problems a computational grid should be adapted to the solution to resolve solution latent features (e.g. wakes, boundary layers, etc.) inherent in real-life applications. Usually, the solution adaptation is an iterative process where grid redefinition (i.e. insertion of new nodes or node movement) happens at each iteration. A flaw in the adaptation algorithm may lead to a locally distorted grid at a given solution-adaptation iteration. Hence, automatic grid adaptation appeals to highly robust discretization schemes, so that it is strongly desirable to understand the behavior of a discretization scheme on distorted grids.

The value $\beta=1.0$ provides a uniform grid, while smaller values of $\beta$ make the grid more distorted. Hence, one may expect a larger solution error, as $\beta$ varies from 
Table 4. Numerical solution to problem (1)-(2). The results of the linear $(K=$ 1) DG discretization for various degrees of distortion $\beta$. The $L_{2}$-norm and the $L_{\infty}$-norm of the solution error are computed on the grid with the number of grid cells $N_{c}=722$.

\begin{tabular}{cccccc}
\hline$\beta$ & 1.0 & 0.8 & 0.6 & 0.4 & 0.2 \\
\hline$e_{L 2}$ & $0.3796 e-3$ & $0.1614 e-2$ & $0.4728 e-2$ & $0.1783 e-1$ & $0.3975 e-1$ \\
$e_{L \infty}$ & $0.7592 e-3$ & $0.5497 e-2$ & $0.2115 e-1$ & $0.8361 e-1$ & $0.1899 e-1$ \\
\hline
\end{tabular}

Table 5. The results of DG discretization for various polynomial degrees $K .0$ in the advection problem. The grid distortion parameter is $\beta=0.5$. The $L_{2}$-norm and the $L_{\infty}$-norm of the solution error are computed on the grid with the number of grid cells $N_{c}=722$.

\begin{tabular}{lcccc}
\hline$K$ & 1 & 2 & 3 & 4 \\
\hline$e_{L 2}$ & $0.9477 e-2$ & $0.7579 e-3$ & $0.3332 e-4$ & $0.2609 e-5$ \\
$e_{L \infty}$ & $0.4141 e-1$ & $0.6943 e-2$ & $0.2182 e-3$ & $0.1954 e-4$ \\
\hline
\end{tabular}

1.0 to smaller values. This conclusion is confirmed by Table 4 where the results of the linear $(K=1)$ DG discretization are presented for various $\beta$.

We also compute the solution error function $e(x, y)$ on each grid cell $e_{i}$ as

$$
e(x, y)=\left|U(x, y)-u_{h}(x, y)\right|, \quad(x, y) \in e_{i}
$$

The solution error distribution over the grid is given in Fig. 2(b). It can be seen from the figure that the grid distortion affects the accuracy of the DG discretization. The maximum solution error appears in the distorted strip of grid cells and it then propagates over the domain once the solution front has passed the distorted region.

The results above demonstrate that grid distortion may slow down the convergence or even result in a divergent numerical solution, as the solution accuracy decreases on distorted grids. The convergence of the numerical solution can be often accelerated by means of a local grid refinement that usually makes a grid smoother in the region of the original distortion. Another way to improve the convergence is iterative $p$-refinement, i.e. increasing of a polynomial degree in a discretization method to resolve the solution. It has been demonstrated in the previous section that the $p$-refinement is not always efficient with a scheme on expanded stencils, as the LS solution reconstruction for higher polynomial degree provided worse results over a distorted grid. Thus, our next test is to increase the $K$ in expansion (10) to verify whether the $p$-refinement will help to resolve the solution in a region of grid distortion. The results for various polynomial degrees in the DG method are presented in Table 5 for the given value $\beta=0.5$. It can be seen from the table that increasing a polynomial degree reduces the solution error leading therefore to a better convergence rate of the approximate solution. 
Finally, we consider a high-order DG discretization as an example of the scheme on compact stencils for a convection-diffusion equation. From a geometry viewpoint, the convection-diffusion problem presents a more difficult case. Namely, a DG discretization of diffusion terms requires a more expanded stencil, as flux balance equations now depend on the neighbor of neighbors cells (see [Cockburn et al. (2000)] for more details).

We consider the following convection-diffusion equation in the unit square $\Omega$

$$
u_{x}+f^{\prime}(x) u_{y}=\mu u_{y y}, \quad(x, y) \in(0,1) \times(0,1)
$$

where the derivative $f^{\prime}(x) \equiv \mathrm{d} f / \mathrm{d} x$ defines a convective velocity, and $\mu$ is a diffusion coefficient.

After the transformation

$$
\xi=\frac{\left(y-y_{0}\right)-f\left(x-x_{0}\right)}{\sqrt{4 \mu\left(x-x_{0}\right)}},
$$

the exact solution to Eq. (18) is given by

$$
u(x, y)=\operatorname{Erf}(\xi)
$$

where the error function $\operatorname{Erf}(z)$ is defined as

$$
\operatorname{Erf}(z)=\frac{2}{\sqrt{\pi}} \int_{0}^{z} \exp \left(-\omega^{2}\right) \mathrm{d} \omega .
$$

The exact solution depends on the shape of the function $f$. The choice of $f(x)$ as

$$
f(x)=\frac{1}{8} \sin 5 x
$$

leads to a "shear layer" solution (see Fig. 3(a)). The origin of a discontinuity in the shear layer is located at $\left(x_{0}, y_{0}\right)$. The shear layer evolution over the domain is characterized by strong local solution gradients which makes this test case attractive for our study.

The boundary conditions for Eq. (18) are chosen to provide a required analytical solution to the problem. Namely, we take

$$
u(0, y)=\operatorname{Erf}(\xi(0, y)), \quad u(x, 0)=\operatorname{Erf}(\xi(x, 0)) \quad \text { and } \quad u(x, 1)=\operatorname{Erf}(\xi(x, 1)),
$$

where the function $\xi(x, y)$ is given by (19).

Let us now briefly comment on a high-order DG discretization used to obtain a numerical solution to problem (18)-(21). The standard approach in discretization of diffusion terms by a DG method is that the equation should be reduced to a 


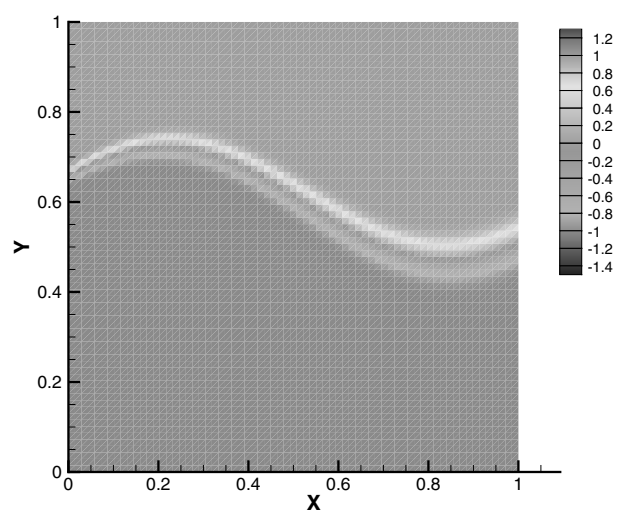

(a)

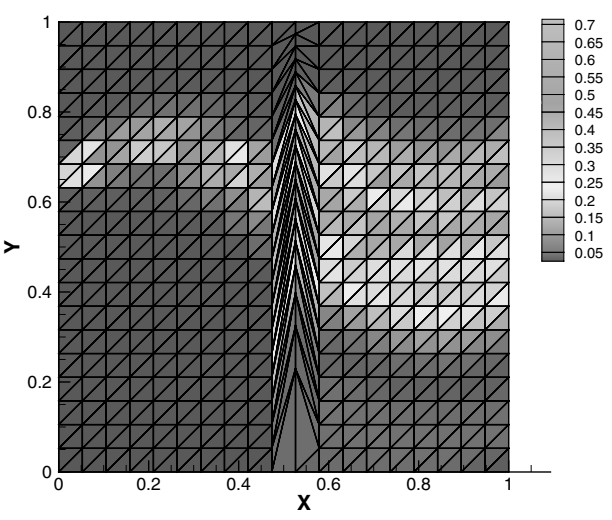

(b)

Fig. 3. (a) The shear layer solution to problem (18)-(21). (b) The distribution of the solution error (17) for problem (18) and (21) over the distorted grid. The number of grid cells $N_{c}=722$ and the distortion parameter $\beta=0.5$.

system of two first-order equations. Introducing a new variable $q=u_{y}$ we arrive at the following system of equations:

$$
\left\{\begin{array}{l}
u_{x}+f^{\prime}(x) u_{y}=\mu q_{y} \\
q=u_{y}
\end{array}\right.
$$

The variable $q(x, y)$ is approximated by using the same basis functions,

$$
q_{h}(x, y)=\sum_{m=0}^{M} q_{m} \phi_{m}(x, y), \quad m=0,1, \ldots, M, \quad x, y \in e_{i}
$$

Approximation of the variable $q$ in the equations above requires to determine viscous numerical fluxes $h_{u q}\left(q^{-}, q^{+}\right)=\frac{1}{2}\left(q^{-}+q^{+}\right)$and $h_{q q}\left(u^{-}, u^{+}\right)=\frac{1}{2}\left(u^{-}+u^{+}\right)$in the problem as well as an inviscid flux (11). After the numerical fluxes are defined, the DG discretization scheme reads

$$
\begin{aligned}
-\iint_{e_{j}} u_{h} & \left(\frac{\partial \phi_{l}}{\partial x}+f^{\prime}(x) \frac{\partial \phi_{l}}{\partial y}\right) \mathrm{d} x \mathrm{~d} y+\oint_{\partial e_{i}} h_{u}\left(u^{+}, u^{-}\right) \phi_{l}\left(n_{x}+f^{\prime}(x) n_{y}\right) \mathrm{d} s \\
=- & \mu \iint_{e_{j}} q_{h} \frac{\partial \phi_{l}}{\partial y} \mathrm{~d} x \mathrm{~d} y+\mu \oint_{\partial e_{i}} h_{u q}\left(q^{+}, q^{-}\right) \phi_{l} n_{y} \mathrm{~d} s, \\
& \iint_{e_{j}} q_{h} \phi_{l} \mathrm{~d} x \mathrm{~d} y=-\iint_{e_{j}} u_{h} \frac{\partial \phi_{l}}{\partial y} \mathrm{~d} x \mathrm{~d} y+\oint_{\partial e_{i}} h_{q q}\left(u^{+}, u^{-}\right) \phi_{l} n_{y} \mathrm{~d} s .
\end{aligned}
$$

In our numerical experiments we choose $x_{0}=-0.1$ and $y_{0}=0.6$. The diffusion coefficient is $\mu=0.5 \cdot 10^{-3}$ to provide a sharp shear layer propagating over the domain. Similarly to a pure advection case, we measure the $L_{2}$-norm and the $L_{\infty}$-norm of the solution error on distorted grids generated for various values of $\beta$. 
Table 6. Numerical solution to the "shear layer" problem (18)-(21). The results of the linear $(K=1)$ DG discretization for various degrees of distortion $\beta$. The $L_{2}$-norm and the $L_{\infty}$-norm of the solution error are computed on the grid with the number of grid cells $N_{c}=722$.

\begin{tabular}{cccccc}
\hline$\beta$ & 1.0 & 0.8 & 0.6 & 0.4 & 0.2 \\
\hline$e_{L 2}$ & $0.5421 e-1$ & $0.7341 e-1$ & 0.1278 & 0.18734 & 0.2558 \\
$e_{L \infty}$ & 0.2785 & 0.4163 & 0.6986 & 0.9169 & 0.9457 \\
\hline
\end{tabular}

Table 7. The shear layer problem. The results of the DG discretization for various polynomial degrees $K>0$. The grid distortion parameter is $\beta=0.5$. The $L_{2}$-norm and the $L_{\infty}$-norm of the solution error are computed on the grid with the number of grid cells $N_{c}=722$.

\begin{tabular}{ccccc}
\hline$K$ & 1 & 2 & 3 & 4 \\
\hline$e_{L 2}$ & 0.1574 & 0.1011 & $0.6533 e-1$ & $0.4267 e-1$ \\
$e_{L \infty}$ & 0.8143 & 0.5896 & 0.4186 & 0.3014 \\
\hline
\end{tabular}

The results of the linear DG discretization are presented in Table 6. It can be seen from the table that a solution to the convection-diffusion problem is more sensitive to the grid distortion than a pure convection case as a larger solution error appear on a grid with the same degree of distortion. This conclusion is also confirmed by solution error function (17) over the grid displayed in Fig. 3(b). Thus we verify in our next series of computations that increasing a polynomial degree of the discretization leads to a smaller solution error. The results for various polynomial degrees presented in Table 7 for the given value $\beta=0.5$ confirm the robustness of a high-order DG scheme.

\section{Concluding Remarks}

In our paper we have discussed the impact of grid geometry on the results of a highorder discretization. It has been demonstrated that a scheme on a compact stencil is less sensitive to the grid distortion than a scheme on an expanded stencil. Our numerical experience with a high-order discretization on compact stencils for a pure convection and a convection-diffusion equation has showed that the discretization remains robust on grids with highly stretched cells.

Our study can be considered as a first step on the way of understanding the features of high-order discretizations on unstructured grids with arbitrary geometry of grid cells. That may help to answer a crucial question of the modern CFD: whether it is possible to develop an efficient high-order discretization on adaptive unstructured grids? It is now a growing understanding in aerodynamic community that coupling a high-order discretization with grid adaptation to the solution could bring great benefits to the flow simulations [Venkatakrishnan et al. (2003)] 
and we believe that the study of the geometry impact on the quality of a discretization is an essential part for development of future CFD codes for real-life applications.

The priority of the future work should be given to the study of a nonlinear case, as the question still remains open whether a discretization scheme will benefit from the compact stencil in case that nonlinear equations are discretized. There are several issues of importance, however, that should be investigated for linear equations as well. One of them is a choice of basis functions for a high-order FE scheme, as a transformation of the basis (i.e. the alignment of basis functions with the solution gradient) may help to render a discretization more robust. Local increasing of a polynomial degree to resolve the solution on stretched cells is another issue of interest as distorted grids are concerned. Finally, a general question of the geometry study is: how a grid distortion can be recognized for a given discretization procedure? This issue is of utmost importance with regard to the impact of grid geometry on the results of a discretization.

\section{Acknowledgments}

This research was partially supported by the Boeing Company under contracts $101 \mathrm{AB}$ and 104AE, and the Deans' Funding Initiative at University of Birmingham. The authors gratefully acknowledge useful discussions with S. V. Lyapunov. The authors also thank L. B. Wigton for drawing their attention to the convectiondiffusion test case.

\section{References}

Agarwal, R. K. and Halt, D. W. [1999] A compact high-order unstructured grids method for the solution of Euler equations, Int. J. Num. Meth. Fluids. 31, 121-147.

Barth, T. and Frederickson, P. [1990] Higher-order solution of the Euler equations on unstructured grids using quadratic reconstruction, AIAA 90-0013.

Barth, T. J. [1998] Numerical methods for gasdynamic systems on unstructured meshes, Lecture Notes in Computer Science and Engineering 8, 195-284.

Bassi, F. and Rebay, S. [1997] A high-order accurate discontinuous finite element method for the numerical solution of the compressile Navier-Stokes equations, J. Comput. Phys. 131(2), 267-279.

Cockburn, B., Karniadakis, G. E. and Shu, C.-W. [2000] The development of discontinuous Galerkin methods, Lecture Notes in Computer Science and Engineering 11, 3-50.

Coirier, W. J. [1994] An adaptively refined, cartesian, cell-based scheme for the Euler and Navier-Stokes equations, PhD Thesis, University of Michigan, USA.

Darmofal D. L. and Haimes R. [2005] Towards the next generation in CFD, AIAA 2005$008 \%$.

Hughes, T. J. R. and Brooks, A. [1979] A multidimensional upwind scheme with no crosswind diffusion, Finite Element Methods for Convection Dominated Flows, ASME, New York.

Mavriplis, D. J. [2003] Revisiting the least-square procedure for gradient reconstruction on unstructured meshes, AIAA 2003-3986. 
Ollivier-Gooch, C. and Van Altena, M. [2002] A high-order-accurate unstructured mesh finite-volume scheme for the advection-diffusion equation, J. Comp. Phys. 181, 729-752.

Petrovskaya, N. B. [2001] Modification of a finite volume scheme for Laplace's equation, SIAM J. Sci. Comput. 23(3), 891-909.

Petrovskaya, N. B. [2004] The choice of weight coefficients for the least-squares gradient approximation, J. Math. Mod. 16(5), 83-93.

Sherwin, S. J. and Peiro, J. [2002] Mesh generation in curvilinear domains using high-order elements, Int. J. Num. Meth. Engrg. 53, 207-223.

Venkatakrishnan, V., Allmaras, S., Kamenetskii, D. and Johnson, F. [2003] Higher order schemes for the compressible Navier-Stokes Equations, AIAA 2003-398\%.

Vigneron, D., Vaassen, J.-M. and Essers, J. A. [2003] An implicit high order cell-centered finite volume scheme for the solution of three-dimensional Navier-Stokes equations on unstructured grids, Third MIT Conference on Computational Fluid and Solid Mechan$i c s$, MA, USA. 